\title{
Revisiting Integer Factorization using Closed Timelike Curves
}

\author{
Soumik Ghosh ${ }^{1}$, Arnab Adhikary ${ }^{2}$ and Goutam Paul ${ }^{3}$ \\ ${ }^{1}$ Institute for Quantum Computing, \\ University of Waterloo, ON N2L 3G1, Canada. \\ Email: soumik.ghosh@uwaterloo.ca \\ ${ }^{2}$ Centre for High Energy Physics, \\ Department of Physics, \\ Indian Institute of Science, Bangalore 560 012, India. \\ Email: arnaba@iisc.ac.in \\ ${ }^{3}$ Cryptology and Security Research Unit, \\ R. C. Bose Centre for Cryptology and Security, \\ Indian Statistical Institute, Kolkata 700 108, India. \\ Email: goutam.paul@isical.ac.in
}

\begin{abstract}
Closed Timelike Curves are relativistically valid objects allowing time travel to the past. Treating them as computational objects opens the door to a wide range of results which cannot be achieved using non relativistic quantum mechanics. Recently, research in classical and quantum computation has focused on effectively harnessing the power of these curves. In particular, Brun (Found. Phys. Lett., 2003) has shown that CTCs can be utilized to efficiently solve problems like factoring and QSAT (Quantified Satisfiability Problem). In this paper, we find a flaw in Brun's algorithm and propose a modified algorithm to circumvent the flaw.
\end{abstract}

\section{INTRODUCTION}

Closed Timelike Curves (CTCs) arise as one of the admissible solutions [1-3] to Einsteins field equations of the general theory of relativity. Although not proven to exist, they opened the doors for possible time travel to the past and assorted paradoxes. Ever since, physicists [4-6] have pondered over their ramifications and causal violations. Their existence was thought to be highly implausible as they seemed to violate the chronology protection conjecture [7]. The situation changed when David Deutsch, in his revolutionary paper [8], imposed a selfconsistency condition for CTCs, resolving the causal violations and giving scientists an information theoretic tool to study such curves. His model for CTCs became popularly known as Deutschian Closed Timelike Curves (DCTCs). Later, Bennett and Schumacher [9] suggested an alternative nonequivalent formulation of CTCs using quantum teleportation and post-selection (P-CTCs). This was further developed by Seth Lloyd [10] and experimentally simulated [11] by Aephraim Steinberg's group.

In recent years, a new branch of research has sprung up to fathom the implications of such curves, modelling them with tools from information theory. They have been shown to possess enormous computing power and can lead to counter-intuitive solutions to complex com-

Part of this work was done while the first two authors were visiting R. C. Bose Centre for Cryptology and Security, Indian Statistical Institute, Kolkata during the Summer of 2017 (between the 6th and the 7th semesters of their Bachelor of Engineering course in Electronics \& Telecommunication Engineering Department of Jadavpur University) for internship under the supervision of the third author. putational problems. They are particularly interesting as they allow non-linear quantum mechanics [12-14], which has interesting ramifications.

Dave Bacon [15] showed that a quantum computer with access to D-CTCs can solve NP-complete problems using only polynomial number of computations. Scott Aaronson [16] showed that if D-CTCs are part of reality, quantum computers are no more computationally efficient than classical Turing machines.

Brun [17] showed a quantum computer equipped with a D-CTC can detect non orthogonal states. There were also been efforts to clone quantum states [18] in the presence of CTCs. DeJonghe et al. [19] proved the evolution of the chronology respecting part of a D-CTC can be a discontinuous function of the initial state. Pati et al. [20] showed it was not possible to purify mixed states of qubits traversing a D-CTC while still being consistent. The results of Brun et al. [17] imply D-CTCs can break the Holveo [21] bound and violate the uncertainty principle. Bennett et al. [22] has questioned some of these striking results, suggesting the circuits of $[16,17]$ do not work [23] when acting on a classically labeled mixture of states.

More recent papers by [24] have studied CTCs using the Heisenberg picture. It has also been shown that a density matrix formulation [25] is not valid for a nonlinear theory. P-CTCs have also been extensively studied, their advantage being the fact that they are consistent with path integral [11] formulations. By invoking Aaronsons's result [26], it has been shown [11] that the computational power of P-CTCs is equivalent to that of the complexity class PP. Aaronson's recent work [27] has aimed at understanding what is computable by a CTC.

Additionally, recent works [28-30] have aimed at simulating closed timelike curves and replicating their re- 
sults. There has also been significant research on flow of information [31, 32], causality considerations [33-37], no-go theorems [38], state preparation [39], and thermodynamic considerations [40] within CTCs. CTCs and entanglement [41-44] is an equally interesting avenue for research.

\section{CTCS AND FACTORIZATION}

Before the flurry of works in Closed Timelike Curves, Todd Brun [45] developed an algorithm that would allow us to find out factors of a number in constant time using CTCs. In the same paper, he extended the general framework of his algorithm to solve NP-Hard problems like Quantified Satisfiability Problem (QSAT). The same framework was later used by him in his study of P-CTCs [46]. In this paper, we study the framework and mainly build on Brun's work.

An important remark here is that Brun [45] never explicitly mentioned which type of Closed Timelike Curve he used in his computations. This is a significant issue in his paper [45] which is also mentioned by Aaronson and Watrous [16]. However, we have noted the prevalence of model-agnostic approaches like Brun's in our later sections.

Brun uses paradoxes to construct his arguments as to why the algorithms work. It can't be a Deutschian model [8] as a straightforward grandfather paradox is not enough to cause a contradiction in the Deutschian model. Brun's model [45] for CTCs only requires selfconsistency to be preserved at all costs which is more in line with P-CTCs.

\section{A. Brun's Factoring Algorithm}

An example of using CTCs to factor numbers was proposed by Todd Brun in [45], given in Algorithm 1.

\section{B. Working of the algorithm}

The algorithm has a register timeRegister whose value can be set from the future to a particular integer. Initially, the variable is initialized to 1 . The time instance is stored in $t$. Initially, as the condition of line 6 is not satisfied, the algorithm proceeds from line 8 to line 14 .

By now, a factor of the input is stored in $p$. If the input is a prime number, $p$ stores the number itself. To generalize, $p$ stores the result of factorization.

\section{Use of a $C T C$}

After storing the result in $p$, the CTC is used to alter the value of the variable timeRegister from the future. In line 15 , timeSet $(t, p)$ changes the value of the

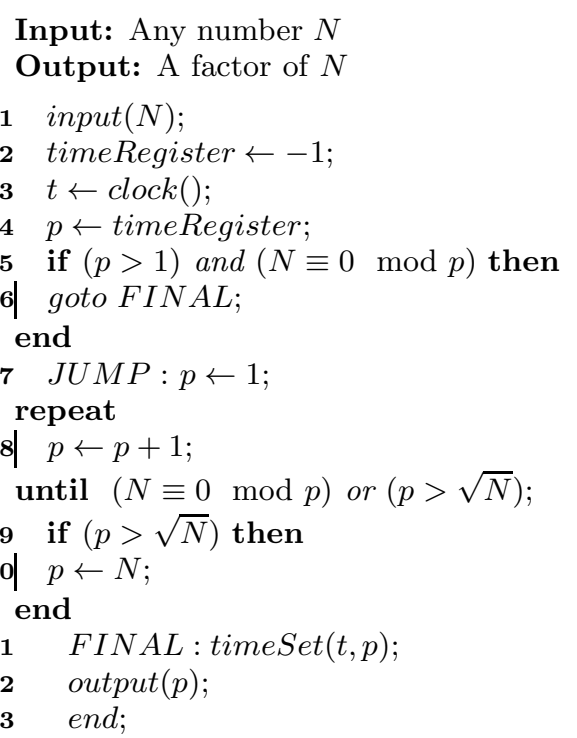

Algorithm 1: Factoring algorithm as proposed by [45]

timeRegister at time instance $t$ to $p$. This has interesting ramifications.

Since, at time instance $t$, the value of timeRegister contains the result, it now satisfies the condition of line 5, as $N$ is now divisible by $p$. Thus, the algorithm branches to line 15. Thereafter, it displays the output $p$.

The algorithm doesn't actually traverse the repeat loop even once. By clever use of a CTC and by threatening to produce a contradiction if the repeat loop is traversed, the algorithm has the result stored in $p$ from time instance $t$ itself. Without a CTC, one needs to iterate through the entire computational block, as is done usually, before arriving at the result.

The same strategy can be utilized for other difficult computations. By going back in time and storing the result of the computation in a special variable, we can ensure that the actual computational block is avoided. This solution, though non-intuitive, is perfectly selfconsistent.

\section{The flaw in the algorithm}

The main claim of the author is that a consistent solution to the problem will mean that the variable timeRegister will contain the factor of the number, before the execution of the loop. This in turn means that the inner loop won't be executed, giving us the factor as soon as the program is run. This is the only selfconsistent situation although quite counter-intuitive.

However, there can be another consistent solution. The variable timeRegister, instead of storing a non-trivial factor of the number, can store instead the number itself. Hence, there is no surety that the algorithm will give us the result we desire once it is executed.

The algorithm can work if we modify the if condition in line 5 to reject the cases where $p$ is equal to $N$. But 
then, the algorithm will not work for prime numbers. For primes, in absence of other factors, $p$ has to store $N$.

Basically, the fact that a non-trivial prime factor is found in constant time by just executing the algorithm means that we have effectively erased the process of finding that prime factor, making the output variable free not to take in the desired value of a prime factor.

\section{OUR APPROACH}

While formulating an algorithm using Closed Timelike Curves, care must be taken to avoid spurious solutions which can compromise the working of the algorithm. In the previous section, we have pointed out one such spurious solution in Brun's formulation of the factoring algorithm. Hereafter, we have modified it in a way such that the problem is resolved.

First, we have explained a flaw in the original algorithm. Then, we have put forward a better solution but with more time complexity. After proposing our solution, we have optimized the solution by introducing one more temporal variable. We have taken a model-agnostic approach whose relevance is discussed in the subsection below.

\section{A. Importance of our model-agnostic approach}

It is true that the works of Brun [45] have been superseded by that of Aaronson and Watrous [16] who rigorously studied closed timelike curves in the light of complexity theory. However, there are a few differences between their work and ours.

Primarily, they have based their work on D-CTCs whereas, as stated earlier, the time machine Brun used here is more in line with a model agnostic approach. Such an approach is present in many other works like [35, 47] to name a few. Interestingly, [47] came after [16] published his results.

Evidently, even without assuming a model, one can find interesting qualitative results with regard to complexity and universality in computation by incorporating elements of time travel involved, as has been done by $[47,48]$. This is why Bruns paper [45] might be relevant even today and why the flaw in his original algorithm needs to be rectified and future researchers warned of the common traps and mistakes.

Additionally, while Aaronson's work contends that closed timelike curves equip both quantum and classical computers with the power of solving PSPACE problems, we are concerned with a particular instance of the complexity class PSPACE, namely, a factoring algorithm. Even after acknowledging the power of classical computers to utilize closed timelike curves in solving problems belonging to PSPACE, and the well-known class NP contained in PSPACE, it is still interesting to understand how it does so, which is achieved by studying the factoring algorithm, which is in NP, in detail. Our approach can be utilized in formulating effective algorithms using CTCs for similar problems in NP, without error.

Moreover, from Aaronson's perspective [26, 49], his research and that of others, detailing the astonishing prowess of a computer equipped with D-CTCs, can be used as evidence against the existence of CTCs that follow Deutsch's prescription. This has resulted in many nonequivalent formulations of CTCs, from P-CTCs $[10,11]$ to recent works on other models [50]. For a general approach to study the flow of causality in these curves, a model agnostic approach can yet be relevant.

\section{A MODIFIED BUT INEFFICIENT ALGORITHM}

We can modify the previous algorithm as shown, with the help of one more conditional branch. The modified algorithm is presented as Algorithm 2.

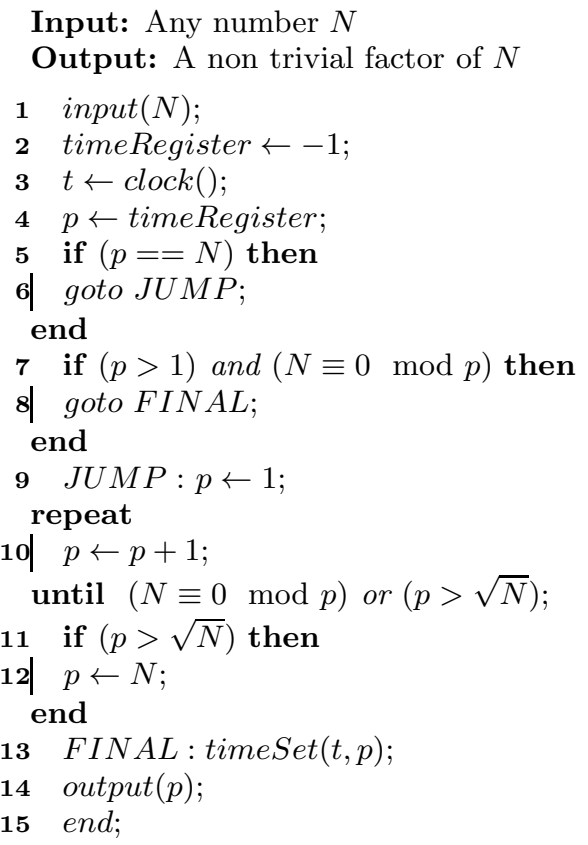

Algorithm 2: Modified factoring algorithm

\section{A. Discussion on correctness}

In this algorithm, we keep an additional check to determine whether $p=N$. If, it is true, then, we force the algorithm to execute the computational block from line 11. If the number is composite, the block spits out a factor of the number which goes back in time via timeRegister to modify the variable $p$, making $p$ contain that factor from our chosen instance.

However, if $p$ did contain the factor in the first place, the condition $p=N$ wouldn't have been valid. This 
gives us a contradiction. By threatening to produce this contradiction, we always ensure that the consistent solution for composite numbers is never the number itself but instead, a non-trivial factor of that number.

\section{B. Problem with prime numbers}

As we saw in the last section, for composite numbers, the main computational block would not run. The algorithm would take constant time $O(1)$ to be implemented.

For prime numbers, we force the algorithm to execute the main computational block. However, the computational block from line 11 gives out the number itself. We go back in time and store the number in timeRegister. This produces no contradictions anywhere in algorithm and $p=N$, in the case of prime numbers, is indeed the consistent result.

But, as a trade-off, for every prime number, the computational block will run with a time complexity of $O(\sqrt{N})$. This is because, for a prime number, the block would iterate through lines 12 to 14, checking every number till the square root of the input integer, and then go back in time to set $p=N$. Hence, the if condition is satisfied for line 5 and there is no way to avoid the repeat loop even with access to a CTC. The repeat loop has worst case complexity $O(\sqrt{N})$, which becomes the worst case complexity for the entire program.

In case of the original algorithm, the time complexity, for both prime and composite numbers, was $O(1)$. What we achieve in terms of correctness is countervailed by a lack of efficiency.

\section{AN OPTIMAL ALGORITHM USING CTC}

We can design a better algorithm with $\mathrm{O}(1)$ complexity with a little computational trickery and the use of an extra register whose value can be modified from the future. It is shown in Algorithm 3.

\section{A. Working of the Algorithm}

The challenge is to provide an algorithm which works optimally for both prime and composite numbers. For this, we use two registers which can be accessed from the future, a timeRegister and a flagRegister. In this case, if $N$ is composite but $p$ stores $N$ itself, we threaten to produce the same contradiction as in the previous section.

However, if the number is prime, we force the program to enter the main computational block, where we change the value of the flagRegister from 0 to 1 . The condition of entering the block in the first place was the flag value being 0 . Hence, once we change the value of timeRegister and flagRegister, a contradiction is produced unless the algorithm avoids the main computa-

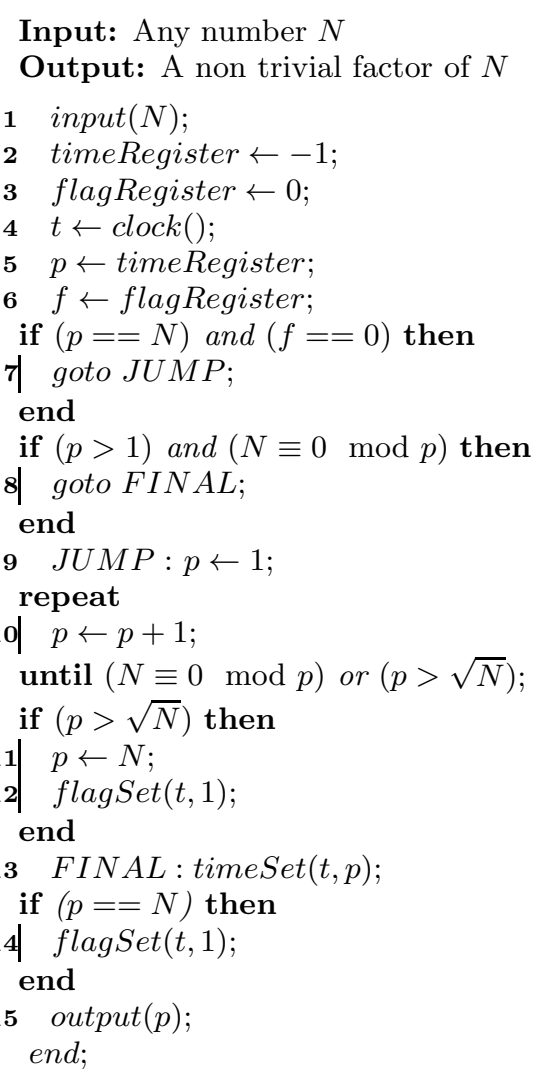

Algorithm 3: Optimal factoring algorithm

tional block for prime numbers, as it did in Brun's original algorithm. This reduces the time complexity for prime numbers to $O(1)$.

For composite numbers, the consistent solution will be $f=0$ and $p=$ factor, while for prime numbers, the consistent solution will be $f=1$ and $p=N$.

\section{CONCLUSION}

In this paper, we point out a flaw in Brun's original factoring algorithm using CTCs [45] and propose a remedy. The modified final algorithm can now easily be used as a recursive tool in factoring large numbers or in factorization in the presence of CTCs with finite length, as Brun has demonstrated in his paper [45].

The case study of the factoring algorithm gives us a caveat to be kept in mind in the future while writing computer codes utilizing self-consistent time travel to the past, as there is always a chance of encountering extraneous fixed points which disturb the actual solution we want to achieve. However, the extraneous fixed points, as have been shown, can be eliminated, at least for the factorization problem, using some more insight. It remains to be seen whether the same holds good for other tasks where a similar issue is encountered. Further research in this domain can focus on how and whether quantum algorithms can speed up by CTCs. Development of a 
[1] Kurt Gödel, An Example of a New Type of Cosmological Solutions of Einstein's Field Equations of Gravitation. Rev. Mod. Phys., 21 447-450, 1949.

[2] W. B. Bonnor, The rigidly rotating relativistic dust cylinder. Journal of Physics A: Mathematical and General, 13(6) 2121, 1980.

[3] J. Richard Gott, Closed timelike curves produced by pairs of moving cosmic strings: Exact solutions. Physical Review Letters., 66(9) 11261129, March 1991.

[4] James B. Hartle, Unitarity and causality in generalized quantum mechanics for nonchronal spacetimes. Physical Review D, 49(12) 65436555, June 1994.

[5] Michael S. Morris, Kip S. Thorne, and Ulvi Yurtsever, Wormholes, time machines, and the weak energy condition. Physical Review Letters, 61(13) 14461449, September 1988.

[6] Kip S. Thorne, Closed Timelike Curves. Proceedings of the 13th International Conference on General Relativity and Gravitation. February 1993.

[7] Stephen Hawking, The chronology protection conjecture. Phys. Rev. D. 603-611, 1992.

[8] D. Deutsch, Quantum mechanics near closed timelike lines. Phys. Rev. D. 44 31973217, 1991.

[9] C. Bennett, talk at QUPON, Wien. http://www.research.ibm.com/people/b/bennetc/, May 2005.

[10] Seth Lloyd, Lorenzo Maccone, Raul Garcia-Patron, Vittorio Giovannetti, Yutaka Shikano, Closed timelike curves via post-selection: theory and experimental demonstration. Phys. Rev. D. 84, 025007) 025007, 2011.

[11] Seth Lloyd, Closed timelike curves via post-selection: theory and experimental demonstration. Phys. Rev. Lett. 106 040403, 2011.

[12] N. Gisin. Weinbergs non-linear quantum mechanics and superluminal communications. Phys. Lett. A, 143 12, 1990.

[13] Daniel S. Abrams (MIT, LNS), Seth Lloyd (MIT), Nonlinear quantum mechanics implies polynomial time solution for NP complete and \#P problems. Phys. Rev. Lett. 81 3992-3995, 1998.

[14] M.J. Cassidy, Nonlinearity in quantum theory and closed timelike curves, Phys. Rev. D52 5676-5680, 1995.

[15] Dave Bacon, Quantum computational complexity in the presence of closed timelike curves. Physical Review A, 70(3) 032309, September 2004.

[16] Scott Aaronson, John Watrous, Closed Timelike Curves Make Quantum and Classical Computing Equivalent, 10.1098/rspa.2008.0350, 2008.

[17] Todd A. Brun, Jim Harrington, and Mark M. Wilde, Localized closed timelike curves can perfectly distinguish quantum states. Physical Review Letters, 102(21) 210402, May 2009.

[18] Ahn, C.R. Myers, T.C. Ralph, R.B. Mann, Quantum state cloning in the presence of a closed timelike curve, Phys. Rev. A 88 022332, 2013.

[19] Richard DeJonghe, Kimberly Frey, and Tom Imbo, Discontinuous quantum evolutions in the presence of closed timelike curves. Physical Review D, 81 087501, 2010.
arXiv:0908.2655

[20] Arun K. Pati, Indranil Chakrabarty, and Pankaj Agrawal, Purification of mixed state with closed timelike curve is not possible. May 2010. arXiv:1003.4221.

[21] Alexander S. Holevo, Bounds for the quantity of information transmitted by a quantum channel. Problems of Information Transmission, 9 177183, 1973.

[22] Charles H. Bennett, Debbie Leung, Graeme Smith, and John A. Smolin, Can closed timelike curves or nonlinear quantum mechanics improve quantum state discrimination or help solve hard problems? Physical Review Letters, 103(17) 170502, October 2009.

[23] Charles H. Bennett, Debbie Leung, Graeme Smith, and John Smolin, The impotence of nonlinearity: Why closed timelike curves and nonlinear quantum mechanics dont improve quantum state discrimination, and havent been shown to dramatically speed up computation, if computation is defined in a natural, adversarial way. Rump Session Presentation at the 13th Workshop on Quantum Information Processing, Zurich, Switzerland, January 2010.

[24] T.C. Ralph, C.R. Myers, Information Flow of quantum states interacting with closed timelike curves. Phys. Rev. A 82 062330, 2010.

[25] Eric G. Cavalcanti and Nicolas C. Menicucci, Verifiable nonlinear quantum evolution implies failure of density matrices to represent proper mixtures. April 2010. arXiv:1004.1219.

[26] Scott Aaronson, Quantum computing, postselection, and probabilistic polynomial-time. Proceedings of the Royal Society A, 461(2063) 34733482, November 2005.

[27] Scott Aaronson, Mohammad Bavarian, Giulio Gueltrini, Computability Theory of Closed Timelike Curves. arXiv:1609.05507, 2016.

[28] Martin Ringbauer, Matthew A. Broome, Casey R. Myers, Andrew G. White, Timothy C. Ralph, Experimental simulation of closed timelike curves, Nature Commun. 4145, 2015.

[29] Todd A. Brun, Mark M. Wilde, Simulations of closed timelike curves, Found. Phys. 47 375-391, 2017.

[30] Xiao Yuan, Syed M. Assad, Jayne Thompson, Jing Yan Haw, Vlatko Vedral, Timothy. C. Ralph, Ping Koy Lam, Christian Weedbrook, and Mile Gu., Replicating the benefits of closed timelike curves without breaking causality. npj Quantum Information, 1:15007, November 2015. arXiv:1412.5596.

[31] Lucas Dunlap, The metaphysics of D-CTCs: On the underlying assumptions of Deutsch's quantum solution to the paradoxes of time travel. Stud. Hist. Phil. Sci. 56 39-47, 2015

[32] Indranil Chakrabarty, Tanumoy Pramanik, Arun K Pati, Pankaj Agrawal CTC assisted PR box type correlation can lead to signaling. Quantum Information and Computation, 14(13) 1251, 2014.

[33] Asutosh Kumar, Indranil Chakrabarty, Arun Kumar Pati, Aditi Sen De, Ujjwal Sen, Quantum no-go theorems in causality respecting systems in presence of closed timelike curves: Tweaking the Deutsch condition. arXiv:1511.08560, 2018. 
[34] Joel J. Wallman and Stephen D. Bartlett, Revisiting consistency conditions for quantum states of systems on closed timelike curves: an epistemic perspective. May 2010. arXiv:1005.2438.

[35] F. Lobo and P. Crawford, Time, Closed Timelike Curves and Causality. The Nature of Time: Geometry, Physics and Perception, pp 289-296 2003.

[36] S M Korotaev and E O Kiktenko, Quantum causality in closed timelike curves. Physica Scripta, 90(8) 2015.

[37] min Baumeler and Stefan Wolf, Non-Causal Computation. Entropy, 19, 3262017.

[38] Sasha Sami, Indranil Chakrabarty Asutosh Kumar, Indranil Chakrabarty, Arun Kumar Pati, Aditi Sen De, Ujjwal Sen, A note on superposition of two unknown states using Deutsch CTC model. Mod. Phys. Lett. A, 31(29) 1650170, 2018.

[39] Arun K. Pati, Indranil Chakrabarty, Pankaj Agrawal, Purification of Mixed State with Closed Timelike Curve is not Possible. Phys. Rev. A, 84, 0623252011.

[40] Magorzata Bartkiewicz, Andrzej Grudka, Ryszard Horodecki, Justyna odyga, Jacek Wychowaniec, Closed timelike curves and the second law of thermodynamics. arXiv:1711.08334, 2017.

[41] Z. Gedik, Eigenvalue Formulation of Quantum Mechanics Near Closed Timelike Curves. arXiv:1201.4870. 2012.

[42] Subhayan Roy Moulick, Prasanta K. Panigrahi, En- tanglement in a World with Closed Timelike Curves. arXiv:1511.00538, 2015.

[43] Eylee Jung, DaeKil Park, Mixedness and Entanglement in the presence of Localized Closed Timelike Curves. arXiv:1506.02333. 2015.

[44] Arun K. Pati, Indranil Chakrabarty, Pankaj Agrawal, Quantum States, Entanglement and Closed Timelike Curves. AIP Conference Proceedings, 1384, 762011.

[45] Todd Brun, Computers with closed timelike curves can solve hard problems. Foundations of Physics Letters, 16 245253, 2003.

[46] Todd A. Brun and Mark M. Wilde, Perfect state distinguishability and computational speedups with postselected closed timelike curves. Found Phys (2012) 42: 341. https://doi.org/10.1007/s10701-011-9601-0

[47] Selim Akl, Time travel: A new hypercomputational paradigm. Int. J. of Unconventional Comp, 6, 329351, 2010.

[48] Hans Moravec, Time travel and computing. https://philpapers.org/rec/MORTTA-2, 1991.

[49] Scott Aaronson. NP-complete problems and physical reality. ACM SIGACT News, 36(1) 30-52, March 2005. arXiv:1412.5596.

[50] John-Mark A. Allen, Treating time travel quantum mechanically. Physical Review A, 90(4) 042107, 2014. arXiv:1401.4933. 